\title{
Aggressive Ciliary Body Adenocarcinoma with Bilateral Lung Metastases: Histological, Molecular, Genetic and Clinical Aspects
}

\author{
Konstantinos Kopsidas ${ }^{a} \quad$ Hardeep Mudhar $^{b}$ Karen Sisley $^{c}$ \\ David W. Hammond $^{c}$ L. Worthington ${ }^{c, d}$ Kim Suvarna $^{e}$ Bernadette Foran ${ }^{f}$ \\ Sachin Salvi ${ }^{a}$ \\ a Sheffield Ocular Oncology Service, Department of Ophthalmology, Royal Hallamshire Hospital, Sheffield, UK; \\ ${ }^{b}$ National Specialist Ophthalmic Pathology Service (NSOPS), Department of Histopathology, Royal Hallamshire \\ Hospital, Sheffield, UK; ${ }^{C}$ Academic Unit of Ophthalmology and Orthoptics, Department of Oncology and \\ Metabolism, University of Sheffield, the Medical School Sheffield, Sheffield, UK; d Sheffield Hallam University, \\ Sheffield, UK; ${ }^{e}$ Department of Histopathology, Royal Hallamshire Hospital, Sheffield, UK; ${ }^{\mathrm{f}}$ Department of Clinical \\ Oncology, Weston Park Hospital, Sheffield Teaching Hospitals NHS Foundation Trust, Sheffield, UK
}

\section{Keywords}

Evisceration · Exenteration · Ciliary body adenocarcinoma · Lung metastasis

\begin{abstract}
Purpose of the Study: To describe the clinical and histopathological features of an aggressive ciliary body adenocarcinoma with pulmonary metastases and skull base spread. Procedures and Results: A 45-year-old female patient presented with a post-traumatic phthisical eye that was eviscerated. This showed an unexpected carcinoma (positive for cytokeratins and melanocytic markers), the histological differential diagnosis for which included a primary ciliary body adenocarcinoma or a metastasis. The patient developed rapid post-surgical localized recurrence that required an orbital exenteration. This showed identical tumour to the evisceration specimen, with vascular invasion in orbital blood vessels and a contaminated orbital soft tissue margin. Staging imaging revealed multiple lung metastases, which were biopsied and shown to be a disseminated ciliary body adenocarcinoma rather than a disseminated primary lung carcinoma. The tumour spread locally to the skull base for which radiother-
\end{abstract}

\section{KARGER}

(c) 2018 S. Karger AG, Basel

E-Mail karger@karger.com

www.karger.com/oop apy was given. Unfortunately, the patient passed away a few weeks later. Conclusions: To our knowledge, this is the first case of ciliary body adenocarcinoma with bilateral lung metastases. The malignant potential of these tumours should be considered as a possibility, and appropriate screening and staging tests should therefore be considered to guide appropriate management.

(c) 2018 S. Karger AG, Basel

\section{Introduction}

Adenocarcinomas of the ciliary body epithelium is exceedingly rare [1-3]. They can be melanotic or amelanotic clinically, have no sex predilection and are often associated with a phthisical eye or underlying chronic inflammation $[3,4]$. The clinical and histopathological features are distinctive enough to differentiate them from other intraocular tumours such as uveal melanoma [5]. Although the most frequent malignant intraocular tumour in phthisical eyes is choroidal melanoma, ciliary body adenocarcinoma should be considered in adults with a longstanding blind eye, especially when associated 
with an epibulbar mass, proptosis, and pain of recent onset [3].

Although its ability for extraocular extension and local spread has been demonstrated previously [3], there are no histologically proven cases of haematogenous metastasis to other sites. We describe a unique case of an aggressive ciliary body adenocarcinoma that was discovered incidentally on an evisceration specimen with bilateral, histologically confirmed lung metastases and local skull base invasion.

\section{Case Report}

A 45-year-old female of South Asian origin presented to her local ophthalmology department with worsening right ocular pain. She had a past history of trauma to her right eye in childhood, resulting in an uncomfortable, blind, phthisical eye. She had also been treated for recurrent right ocular inflammatory and corneal disease over the last 2 years. Her left eye was pseudophakic with an iris and retinochoroidal coloboma. She had previously received antiviral therapy relating to hepatitis $\mathrm{C}$ junior type 3 , with side effects including interferon-induced hypothyroidism and eczema.

Given her worsening symptoms, she underwent right eye evisceration surgery with a 22-mm orbital implant. Preoperative Bscan was not performed. During surgery, the globe contents were deemed to be highly irregular and were sent to histopathology for analysis. Histology showed a partly necrotic pleomorphic, amelanotic carcinoma, immunohistochemically positive for cytokeratins. The differential diagnosis included a primary retinal pigment epithelial or ciliary body adenocarcinoma or metastatic carcinoma. The patient was urgently referred to our oncology service for further management. Examination revealed a tense, inflamed, proptosed right orbit with significant chemosis (Fig. 1a). Palpation of the right orbit demonstrated a hard mass, suggesting extensive recurrence of the adenocarcinoma. A CT and MRI scan of the orbits revealed a large inferolateral, intraorbital soft tissue mass measuring $27 \mathrm{~mm}$, in contact with the inferolateral bony orbit (Fig. 1b, c). There was no evidence of extra-orbital extension or bone erosion. No regional lymphadenopathy was demonstrated.

Given the aggressive progression of the disease after evisceration surgery, an urgent right orbital exenteration surgery was performed for local disease control. The histology showed a malignant tumour, composed of highly pleomorphic, amelanotic epithelial cells with eosinophilic to vacuolated/clear cytoplasm and atypical mitotic figures and necrosis present. No basement membrane elaboration or Alcian blue-positive material was present. The tumour was immunohistochemically positive for pan-cytokeratins (AE1-AE3) and cytokeratin-7, and was negative for Napsin A, Melan A, S100, HMB45, SOX10, CD56, TTF-1, GATA3, CK20, p63, CK14 and CDX-2 (Fig. 2). The differential diagnosis included a poorly differentiated primary amelanotic retinal pigment epithelium (RPE) adenocarcinoma, a primary ciliary body epithelium adenocarcinoma or metastatic carcinoma to the globe. RPE adenocarcinomas tend to co-express melanocytic markers and epithelial markers. The clear cell morphology is well described in ciliary body adenocarcinomas, so if this tumour was a primary, derivation from the ciliary body non-pigmented epithelium was highly favoured.

Cytogenetic analysis and array comparative genomic hybridization (CGH) were performed on freshly sampled tissue and a sample was cultured as detailed previously [6-8]. The tumour was successfully established in culture and both cultured and uncultured specimens shared the same complex genetic alterations, with minor differences likely to relate to heterogeneity within the original sample. Gains of 1q, partial 3p, 3q and focal $8 \mathrm{q}$ were found with loss of $1 \mathrm{p}, 2 \mathrm{p} / \mathrm{q}$, focal deletion $3 \mathrm{p}, 4 \mathrm{p} / \mathrm{q}$, partial $8 \mathrm{q}$, $9 \mathrm{p} / \mathrm{q}, 11 \mathrm{p}, 13$, partial 14 and $15,17 \mathrm{p} / \mathrm{q}, 18$ and 21 were found by a CGH. The chromosome count ranged from 43 to 103 and imbalances often resulted from complex rearrangements. The changes were not consistent with those reported for ciliary body melanomas [6].

A chest-abdomen-pelvis CT scan revealed bilateral lung nodules, the largest in the right lobe measuring $13 \mathrm{~mm}$ in size (Fig. 1d). Due to the suspicion that these were metastases, and considering the rarity of such a presentation, histopathological confirmation was required prior to deciding further management. The patient underwent an urgent CT-guided core needle lung biopsy. The histopathology of the needle core confirmed a high-grade, pleomorphic non-small cell carcinoma, positive for AE1-AE3 (pan-cytokeratins) and negative for Napsin A, TTF1 and p63 (Fig. 2). The morphological and immunohistochemical profile was entirely in keeping with metastatic adenocarcinoma from the globe and therefore confirmed that the globe tumour was a primary, poorly differentiated ciliary body adenocarcinoma.

At follow-up oncology review, adjuvant radiotherapy to the orbit was discussed, but in view of the disseminated disease, palliative systemic treatment in the form of chemotherapy was deemed to be more appropriate. The metastatic disease status and incurability of the situation was explained to the patient. Due to the fact that she lived some distance from our specialist centre, she was referred to her local oncology centre for chemotherapy and ongoing palliative care.

Six weeks following exenteration surgery and whilst waiting for an urgent appointment at the local oncology centre, she was reviewed in the oncology clinic. The surgical wound was completely healed, and the patient had no respiratory symptoms. The only complaint was of vague ache within the right orbital area, but there were no obvious signs of recurrence or infection at this point. A week later, the patient attended the ocular oncology clinic and a small raised, solid subcutaneous lesion had appeared in the inferolateral orbit, attached to the orbital floor. An urgent MRI scan revealed extensive local recurrence of the malignancy with involvement of the orbital post-exenteration cavity, extending into the skull base and mid-face (Fig. 1e). She was seen urgently by the local oncology team to consider palliative platinum-based chemotherapy. However, she had now developed severe headache (despite regular analgesia) as well as nausea and vomiting secondary to the rapid local progression within the skull base recurrence. For this reason, it was decided to proceed first with palliative local radiotherapy ( 30 Gy in 10 fractions given over 2 weeks, $6 \mathrm{MV}$ photons) to try and halt the disease within the head and neck region and improve her symptoms. Despite urgent palliative radiotherapy to the skull base infiltration, the patient rapidly deteriorated secondary to disease progression and died shortly after treatment was given.
Kopsidas/Mudhar/Sisley/Hammond/ Worthington/Suvarna/Foran/Salvi 

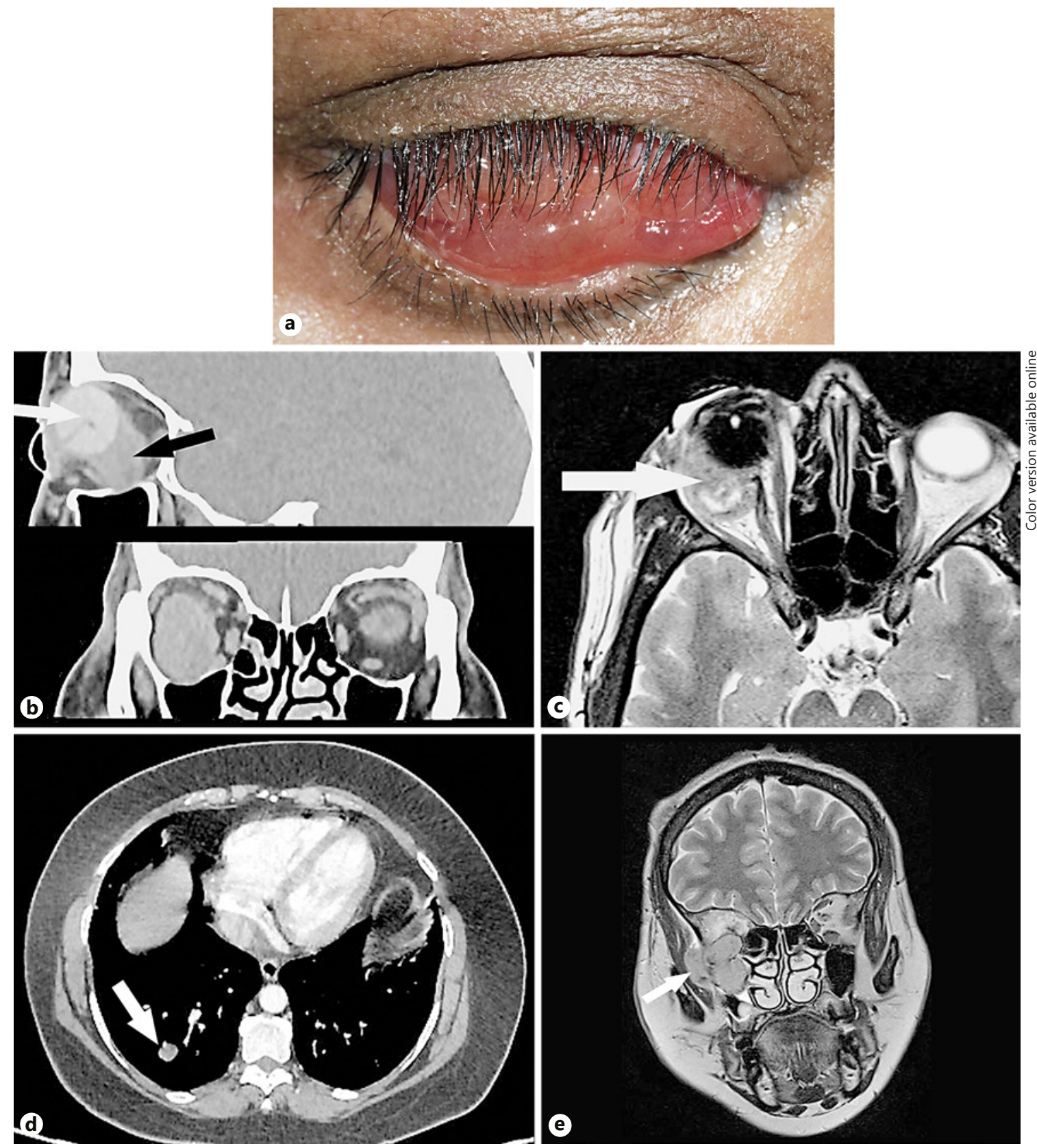

Fig. 1. a External photo from the patient's right orbit following evisceration on presentation. Note the chemosis and orbital swelling. b Sagittal (upper) and coronal (lower) CT scan of the orbits with contrast. Within the orbit on the right side, there is an orbital implant (white arrow) which has been displaced slightly medially and superiorly. Just lateral to this implant, there is a soft tissue mass (black arrow). There is no evidence of any bony erosion as a consequence of this lesion. The left eye and globe do not show any abnormality. c Axial MRI scan of the head and orbits showing a heterogeneously enhancing low T2 signal mass within the right orbit with areas of necrosis centrally (white arrow) representing a recurrence of the previous right ocular adenocarcinoma. This is centred on the inferior lateral quadrant of the orbit, involving the extra- and intraconal spaces. There is no disease beyond the orbital apex and no evidence of bulk invasion into the middle cranial fossa or cavernous sinus. d CT scan of the chest. There are varying-sized nodules in both lungs, the largest of which in the right lower lobe measuring $13 \mathrm{~mm}$ (white arrow). e Sagittal MRI scan of the head and orbits (T2-weighed image) demonstrating a low-enhancing soft tissue mass within the orbital cavity breaching both the orbital floor into the maxillary sinus and also the lateral orbital wall and extending into the masticator space. 


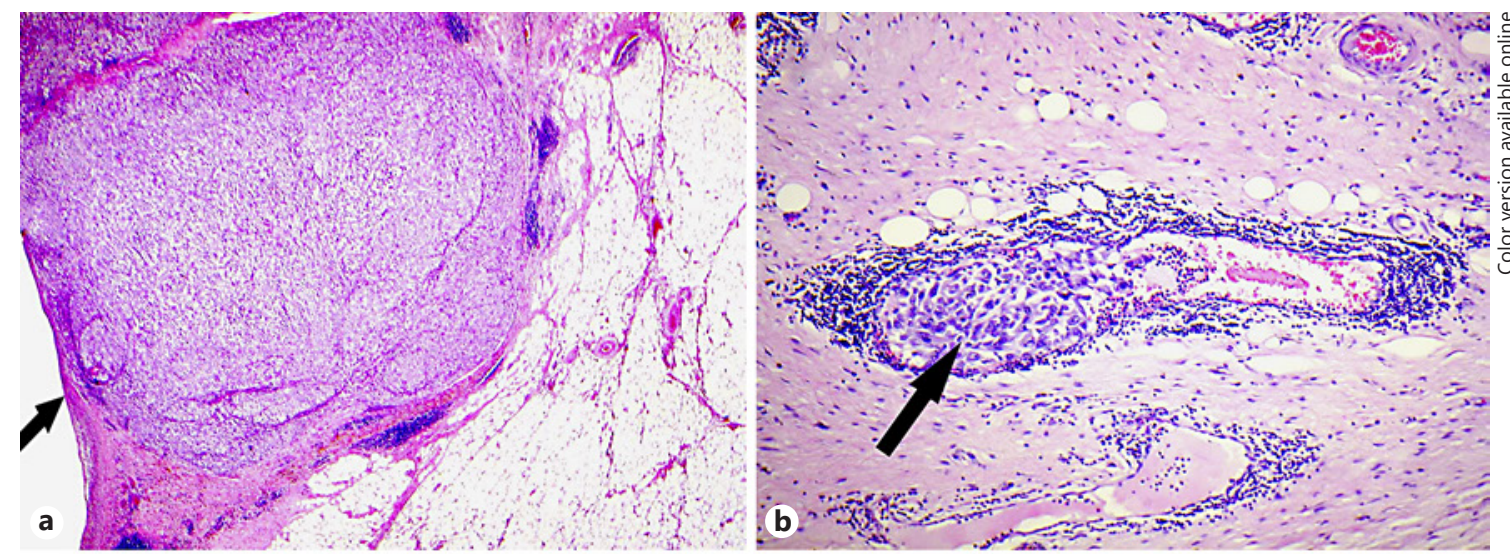

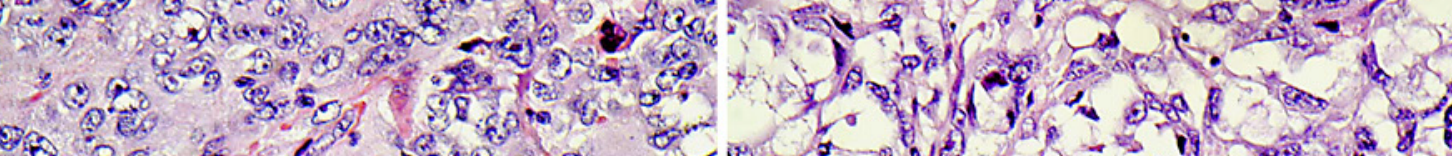

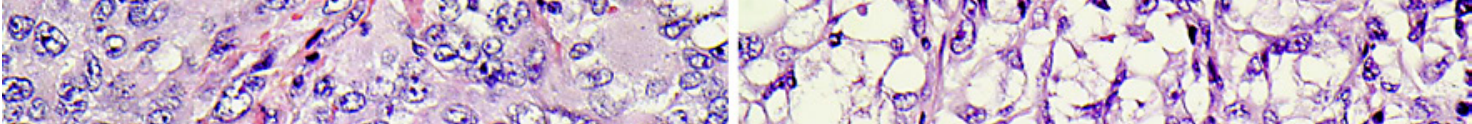

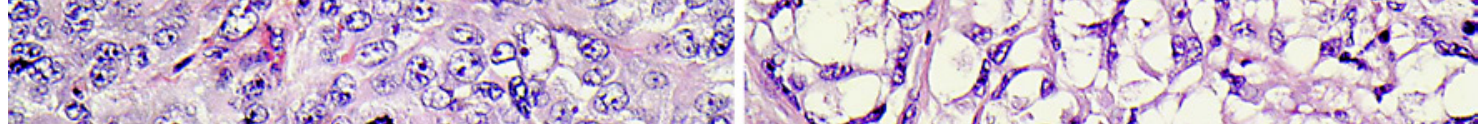

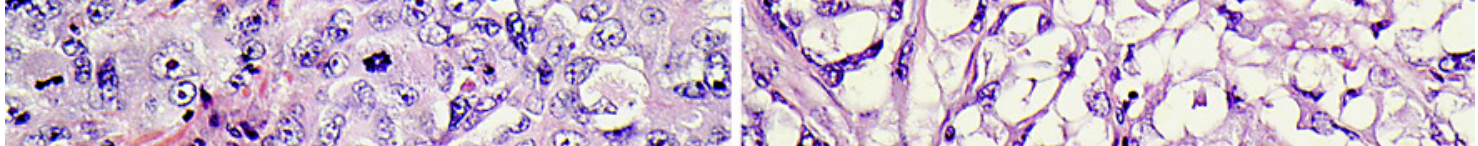

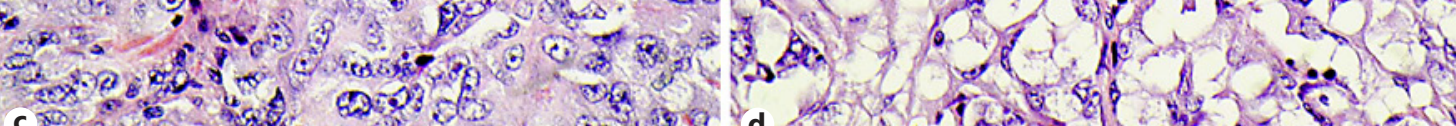

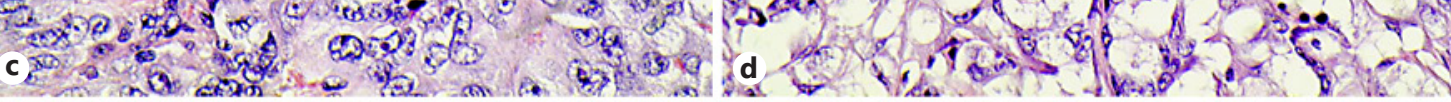

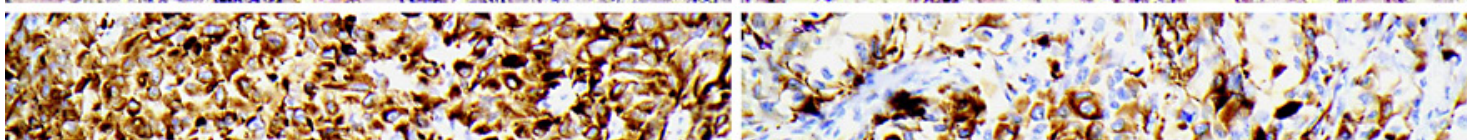

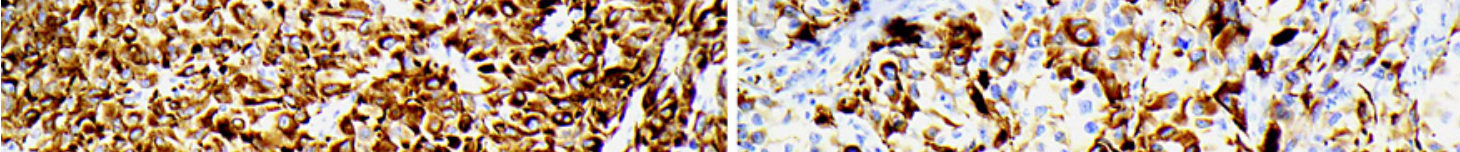

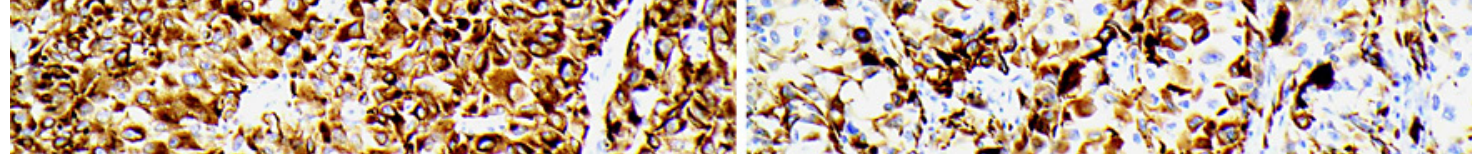

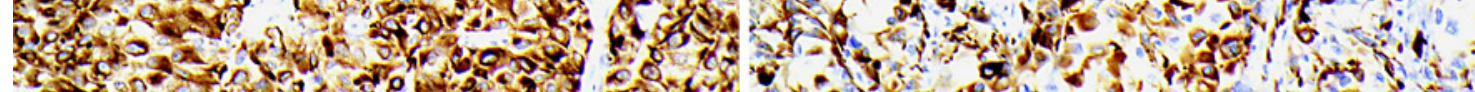

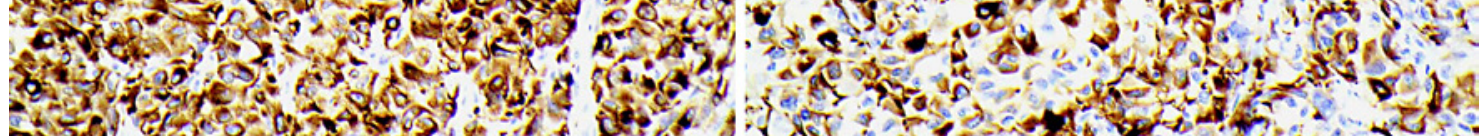

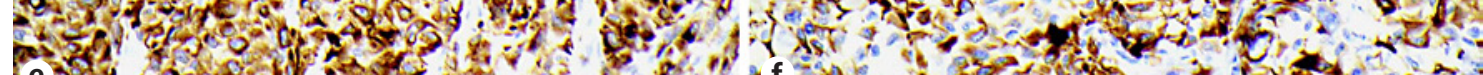

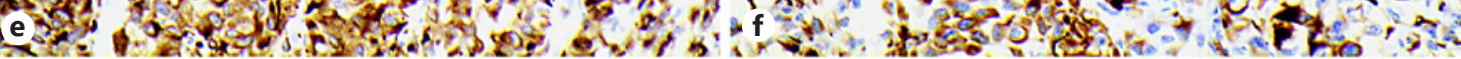

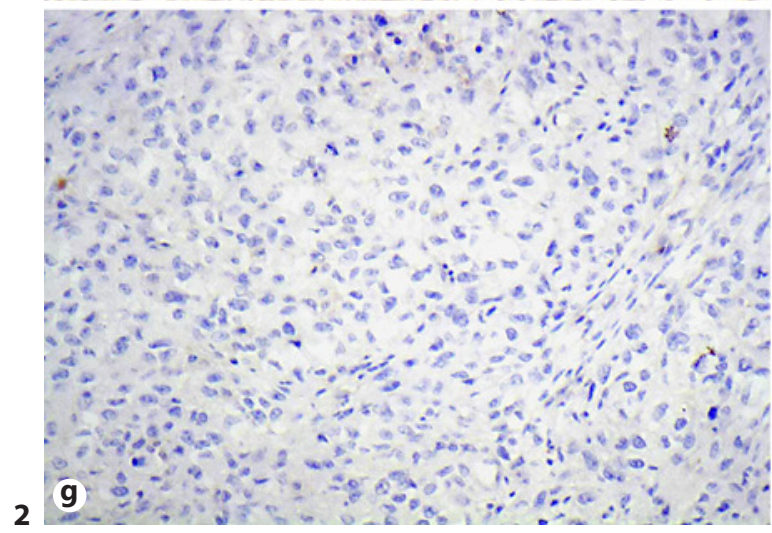

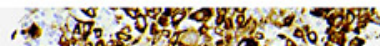

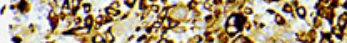

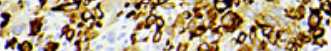

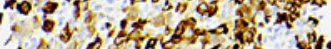

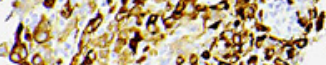
Q

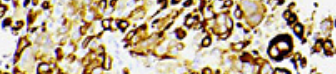

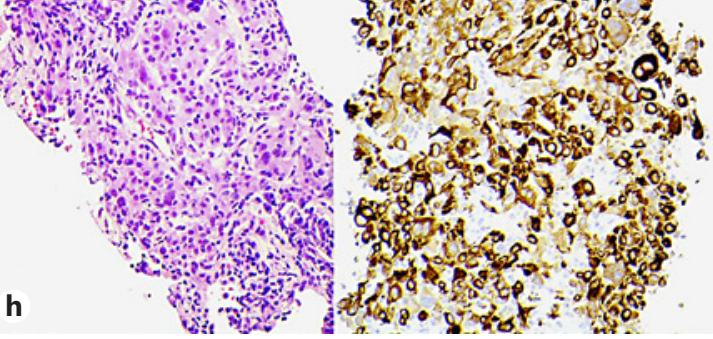

(For legend see next page.)
Ocul Oncol Pathol 2019·5·79-84 DOI: $10.1159 / 000487805$
Kopsidas/Mudhar/Sisley/Hammond/ Worthington/Suvarna/Foran/Salvi 


\section{Discussion}

In the largest series of ciliary body adenocarcinomas published to date, it was noted that 9 out of 12 tumours occurred in phthisical eyes in adults [3]. Tumours that were confined to the globe conferred a favourable prognosis. Extraocular spread was associated with a much worse prognosis, with 3 patients developing direct intracerebral spread and in 2 cases, metastases developed to the parotid gland and to the bone (sacrum and ribs) [3]. In our case, the histological differential diagnosis in the evisceration sample lay between a primary carcinoma of the ciliary body or RPE and a metastatic carcinoma. The presence of prominent basement membrane is a distinctive feature of primary ciliary body epithelial or RPE adenocarcinomas and is never a feature of metastatic carcinomas to the globe [1-4]. In our case, this feature was absent; hence a metastasis had to be excluded. The lung lesions detected on the CT thorax during the staging process triggered a biopsy and confirmed that the tumour was a metastasis from the globe, rather than representing disseminated primary lung carcinoma. Histologically, the tumour was amelanotic and had clear cell change and was negative for SOX10, which are features that favoured its derivation from the ciliary body rather than the more posterior RPE $[9,10]$.

A previous study has examined the cytogenetics of ciliary body adenocarcinoma and found changes in chromosome 6 only. In our case, there were numerous complex gains and losses of several chromosomes that probably reflected the tumour's aggressive cytology and behaviour [11].

The ciliary body adenocarcinoma was initially diagnosed after evisceration for a phthisical, inflamed eye. Evisceration is an established treatment for the management of a painful, blind eye. However, the risk of an ocu-

Fig. 2. a Haematoxylin and eosin image of the exenteration specimen showing the orbital fat being invaded by a nodule of malignant tumour. The black arrow shows the attenuated scleral shell. b Orbital vascular invasion by tumour (arrow). c Ciliary body adenocarcinoma showing eosinophilic, pleomorphic cells, with atypical nuclei and conspicuous nucleoli. $\mathbf{d}$ Ciliary body adenocarcinoma showing distinct cytoplasmic clearing. e The tumour expresses pan-cytokeratins, AE1-AE3 (immunohistochemistry study, brown is positive staining). $\mathbf{f}$ The tumour expresses cytokeratin-7. g The tumour does not express SOX10 (a melanocytic marker). $\mathbf{h}$ The lung core biopsy showing the entire core filled with similar tumour cells (left plate), which express pan-cytokeratin (AE1-AE3) staining (right plate), confirming lung metastases from the ciliary body adenocarcinoma.

Aggressive Ciliary Body Adenocarcinoma with Bilateral Lung Metastases lar tumour having been missed preoperatively could lead to dissemination of tumour cells within the orbit, orbital recurrence, and possible metastasis with subsequent poor prognosis [12]. In a series of 205 eviscerations, Novais et al. [13] found tumours in 4 cases representing $1.95 \%$ of all eviscerations. Eagle et al. [14] reported 7 cases of uveal melanoma detected on evisceration and commented on tumour necrosis mimicking endophthalmitis. Preoperative B-scan as a minimum prior to evisceration surgery for painful blind eyes is always suggested, but because of the fact that preoperative imaging is not always successful for diagnosing a possible intraocular malignancy, the evisceration specimen should always be sent for histological analysis to exclude malignancy, in order to perform prompt adjuvant orbital radiotherapy if necessary [1214]. In our case, according to the evisceration surgical notes, it was noted that the sclera had a linear defect. This may have been due to pre-existing tumour invasion or possibly relate to the childhood trauma. Histologically, it was not possible to make this distinction.

The differential diagnosis of the lung lesions in this case included metastatic ciliary body adenocarcinoma, disseminated primary lung carcinoma or an inflammatory/infectious aetiology. Primary lung tumours are the second most common tumours to metastasize to the eye [15], with the most common histologic type being adenocarcinoma, usually originating in peripheral lung tissue $[16,17]$. Metastasis to the choroid from a lung primary can also be the presenting symptom $[18,19]$. Moreover, of all patients reported to have choroidal metastasis as the presenting symptom of an extraocular primary, the majority (almost 60\%) had lung cancer [20]. Secondly, this patient had a history of treated hepatitis C. Pulmonary sarcoidosis may be observed in hepatitis $\mathrm{C}$ virus patients during or shortly after treatment with antiviral therapy, and it seems to be induced by the interferon $\alpha$ therapy or by the hepatitis $C$ virus infection itself [21].

In cases of metastatic disease, oncologists recommend consideration of systemic therapy with palliative intent chemotherapy to slow down the course of the disease and improve symptom control. The choice of whether to initially proceed with systemic chemotherapy or local radiotherapy is dependent on several patient factors which include: general fitness level, whether symptoms are localized or widespread, previous treatments given and the patient's wishes. Following initial review and discussion after diagnosing lung metastases, the oncology opinion was to proceed with systemic therapy. This was based on the following: the patient was fit, had no localized symptoms but had widespread disease. However, within 3

Ocul Oncol Pathol 2019;5:79-84 DOI: $10.1159 / 000487805$ 
weeks after the lung biopsy and 8 weeks after the exenteration, the patient developed severe localized symptoms within the surgical site and skull base and for this reason the most appropriate course of action was to commence urgent palliative local radiotherapy to the disease within the cavernous sinus region. The radiotherapy was intended to relieve the pain and to halt the local spread within this region. It was felt that this modality would provide more rapid relief of symptoms than chemotherapy at this site. This case demonstrates the need to adapt any planned oncological treatment depending on the patient's evolving disease status and symptoms.

This is the first reported case of histologically confirmed haematogenous metastasis to the lungs from a ciliary body adenocarcinoma, confirming that these tumours have the potential of haematogenous spread, especially if incompletely excised. This specific tumour in our patient seemed to be extremely aggressive, with rapid and extensive local spread even after exenteration surgery. Local spread and systemic metastatic potential should be kept in mind in incompletely excised ciliary body adenocarcinoma and appropriate screening and staging should be performed prior to radical surgery.

This report highlights the importance not only of routine histopathological examination of eviscerated ocular contents and the iatrogenic risks of local spread of the tumour but also the necessity of preoperative imaging such as ocular ultrasound B-scan in chronically blind eyes planned for evisceration.

\section{Statement of Ethics}

The study was performed in accordance with the Declaration of Helsinki. The patient has given informed consent for this case report.

\section{Disclosure Statement}

The authors declare no conflicts of interest or financial interests.

\section{References}

1 Harris JL, Gumucio CC, Ohanion MB: Adenocarcinoma of the ciliary epithelium. Arch Ophthalmol 1960;80:217-219.

2 Dryja TP, Zakov ZN, Albert DM: Adenocarcinoma arising from the epithelium of the iris and ciliary body. Int Ophthalmol Clin 1980; 20:177-190.

3 Laver NM, Hidayat AA, Croxatto JO: Pleomorphic adenocarcinomas of the ciliary epithelium. Immunohistochemical and ultrastructural features of 12 cases. Ophthalmology 1999;106:103-110.

4 Dryja TP, Zakov ZN, Albert DM: Adenocarcinoma arising from the epithelium of the iris and ciliary body. Int Ophthalmol Clin 1980; 20:177-190.

5 Shields JA, Eagle RC, Shields CL, De Potter P: Acquired neoplasms of the nonpigmented ciliary epithelium (adenoma and adenocarcinoma). Ophthalmology 1996;103:2007-2016.

6 Sisley K, Rennie IG, Cottam DW, Potter AM, Potter CW, Rees RC: Cytogenetic findings in six posterior uveal melanomas: involvement of chromosomes 3, 6, and 8. Genes Chromosomes Cancer 1990;2:205-209.

7 Salawu A, Ul-Hassan A, Hammond D, Fernando M, Reed M, Sisley K: High quality genomic copy number data from archival formalin-fixed paraffin-embedded leiomyosarcoma: optimisation of universal linkage system labelling. PLoS One 2012;7:e50415.

8 Hammond DW, Al-Shammari NSD, Danson S, Jacques R, Rennie IG, Sisley K: High-resolution array $\mathrm{CGH}$ analysis identifies regional deletions and amplifications of chromosome 8 in uveal melanoma. Invest Ophthalmol Vis Sci 2015;56:3460-3466.

9 Mori T, Sukeda A, Sekine S, Shibata S, Ryo E, Okano H, Suzuki S, Hiraoka N: SOX10 expression as well as BRAF and GNAQ/11 mutations distinguish pigmented ciliary epithelium neoplasms from uveal melanomas. Invest Ophthalmol Vis Sci 2017;58:54455451.

10 Alkatan HM, Al Qahtani AA, Maktabi AM: Intraocular adenocarcinoma: histopathological report of two cases with different origin. Can J Ophthalmol 2016;51:67-70.

11 Nicolò M, Nicolò G, Zingirian M: Pleomorphic adenocarcinoma of the ciliary epithelium: a clinicopathological, immunohistochemical, ultrastructural, DNA-ploidy and comparative genomic hybridization analysis of an unusual case. Eur J Ophthalmol 2002;12: 319-323.

12 Lemaitre S, Lecler A, Lévy-Gabriel C, Reyes C, Desjardins L, Gentien D, Zmuda M, Jacomet PV, Lumbroso-Le Rouic L, Dendale R, Vincent-Salomon A, Pierron G, Galatoire O, Cassoux NJ: Evisceration and ocular tumors: what are the consequences? Fr Ophtalmol 2017;40:93-101.

13 Novais EA, Fernandes BF, Pacheco LF, Di Cesare S, Miyamoto C, Briccoli TA, Burnier MN Jr: A histopathologic review of undiagnosed neoplasms in 205 evisceration specimens. Ophthal Plast Reconstr Surg 2012;28:331334.
14 Eagle RC Jr, Grossniklaus HE, Syed N, Hogan RN, Lloyd WC 3rd, Folberg R: Inadvertent evisceration of eyes containing uveal melanoma. Arch Ophthalmol 2009;127:141-145.

15 Kreusel KM, Bechrakis N, Wiegel T, Emmerlich T, Foerster MH: Clinical characteristics of choroidal metastasis. Ophthalmologe 2003;100:618-622.

16 Shields CL, Shields JA, Gross NE, Schwartz GP, Lally SE: Survey of 520 eyes with uveal metastases. Ophthalmology 1997;104:12651276.

17 World Cancer Report 2014. Geneva, World Health Organization, 2014, chapt 5.1.

18 Kreusel KM, Wiegel T, Stange M, Bornfeld N, Hinkelbein W, Foerster MH: Choroidal metastasis in disseminated lung cancer: frequency and risk factors. Am J Ophthalmol 2002; 134:445-447.

19 Adulkar N, Radhakrishnan S, Vidhya N, Kim $\mathrm{U}$ : RPE adenocarcinoma as the presenting sign of bronchogenic carcinoma: diagnostic dilemma in the management of a case. Case Rep Ophthalmol Med 2013;2013:786378.

20 Singh A, Singh P, Sahni K, Shukla P, Shukla V, Pant NK: Non-small cell lung cancer presenting with choroidal metastasis as first sign and showing good response to chemotherapy alone: a case report. J Med Case Rep 2010;4:185.

21 Hoffmann RM, Jung MC, Motz R, Gössl C, Emslander HP, Zachoval R, Pape GR: Sarcoidosis associated with interferon-alpha therapy for chronic hepatitis C. J Hepatol 1998;28:1058-1063. 